\title{
Reliability and Validity of Scales for Assessing Child Dental Fear and Anxiety
}

\section{Pouzdanost i valjanost ljestvica za procjenu dentalnog straha $i$ anksioznosti kod djece}

\author{
${ }^{1}$ Private dental office, Slavonski Brod \\ Privatna praksa dentalne medicine, Slavonski Brod \\ 2 Department of Pediatric and Preventive Dentistry, School of Dental Medicine, University of Zagreb, Croatia \\ Zavod za dječju i preventivnu stomatologiju Stomatološkog fakulteta Sveučilišta u Zagrebu
}

\begin{abstract}
Children with high dental anxiety display uncooperative behavior during dental procedures. Different self-assessment questionnaires are in use to estimate the prevalence of dental fear and anxiety (DFA) in children but they need to be tested for reliability and validity in different populations. Objectives: The aim of this study was to test the reliability and validity of two scales for the assessment of child dental fear and anxiety in a sample of Croatian children. Material and Methods: The sample comprised 202 pairs consisting of children and their mothers ( 97 boys and 105 girls) aged $5-12$ years. Two scales were used to assess children's DFA: the Children's Fear Survey Schedule - Dental Subscale (CFSS-DS) and the Modified Child Dental Anxiety Scale - face version (MCDAS-f). The Corah's Dental Anxiety Scale (CDAS) was used in the sample of children's mothers. The average scores of cooperative children and children with behavior management problem (BMP) were compared. Results: Cooperative children displayed significantly lower CFSS-DS scores than children with BMP (M $=28.46$ vs. $M=39.36 ; P<0.001)$. The average MCDAS-f score was significantly higher in children with BMP than in cooperative children $(M=28.07$ vs. $20.01 ; P<0.001)$. The CFSS-DS showed high internal consistency (Cronbach's $\alpha=0.90)$, while internal consistency of MCDAS-f was good $(\alpha=0$. 73). The maternal CDAS showed good internal consistency $(\alpha=0,89)$. The correlations with other self-report measurements of DFS show good validity of all scales. The correlations between CFSS-DS and MCDAS- $f$ were highly significant $(r=0.482 ; P<0.01)$ as well as the correlations of CFSS-DS and MCDAS- $f$ with CDAS scores $(r=0.223 ; P<0.01$ and $r=0.198 ; P<0.01$, respectively. Conclusion: The obtained results suggest that both the CFSS-DS scale and the MCDAS-f scale are reliable and valid scales for measuring dental fear and anxiety in children in a sample of Croatian children. The MCDAS-f scale has shown slightly lower internal consistency but it is suitable for use in young children. In addition, the MCDAS-f scale is simple to administer.
\end{abstract}

Received: December 17, 2019

Accepted: February 2, 2020

Address for correspondence Dr. Tomislav Škrinjarić University of Zagreb School of Dental Medicine Gunduliceva 5, 10000 Zagreb, Croatia.

tskrinjaric@sfzg.hr

\section{Introduction}

Dental treatment of children is frequently associated with anxiety and uncooperative behavior. Dental fear and anxiety (DFA) in children is widespread and its prevalence ranges from $6-20 \%$ (1). Different instruments have been used to assess DFA in children. The commonly used scale that was primarily designed to measure DFA in adults $(2,3)$ is the Corah Dental Anxiety Scale (CDAS). Additionally, this instrument has been successfully used in many studies with children (4-6). The CDAS has been shown to be a reliable and stable scale. Besides, a significant relationship between the CDAS and the Frankl's Rating Scale has been observed (7).

The Children's Fear Survey Schedule - Dental Subscale (CFSS-DS) was a scale especially constructed for use in children (8). This instrument has been widely used in children. The CFSS-DS has been studied in many countries and
Uvod

Stomatološko liječenje djece često je povezano s anksioznošću i nekooperativnim ponašanjem. Kod djece su dentalni strah i anksioznost (DFA) rašireni i njihova se prevalencija kreće od 6 do $20 \%$ (1). Za procjenu DFA-e kod djece korišteni su različiti mjerni instrumenti. Često se primjenjuje ljestvica Corah Dental Anxiety (CDAS), primarno konstruirana za mjerenje DFA-e kod odraslih $(2,3)$. Taj je instrument također uspješno korišten u mnogobrojnim studijama u kojima su ispitanici bila djeca $(4-6)$. CDAS je pouzdan i pokazuje dobru stabilnost te značajnu povezanost $s$ Franklovom ljestvicom ponašanja (7).

Za djecu je posebno izrađena ljestvica Children's Fear Survey Schedule - Dental Subscale (CFSS-DS) (8). Taj se instrument često primjenjuje $\mathrm{u}$ ispitivanjima djece. Testiran je u mnogim zemljama i pokazao je dobru unutarnju pouzda- 
showed good internal reliability and validity (9-12). Cultural differences may play an important role in the reliability of similar scales for the assessment of DFA in children $(11,13$, 14).

Wong et al. (15) developed the Modified Child Dental Anxiety Scale (MCDAS) for the use with 8- to 15-year-old children. This scale is based on the Corah Dental Anxiety Scale (CDAS) and it includes eight questions to assess anxiety in different dental situations. A five-point Likert scale is used to score the level of dental anxiety $(15,16)$. However, the (MCDAS) scale is not suitable for the assessment of dental anxiety in very young children who are not capable of good cognitive functioning.

Howard and Freeman (13) created the Modified Child Dental Anxiety Scale; - Faces Version (MCDAS-f), by modifying the MCDAS by adding of faces analogue scale to the original numeric form. The inclusion of faces to the corresponding numbers allows young and nervous children good cognitive functioning and proper assessment of dental anxiety. The evaluation of psychometric properties of MCDAS-f confirmed that it as a reliable measure of dental anxiety in children (13). Since such scales have to be tested for reliability and validity in different populations and cultures, the aim of this study was to assess the reliability and validity of the CFSS-DS and the MCDAS-f in a sample of Croatian children.

\section{Material and methods}

\section{The sample and its structure}

An assessment of dental anxiety was carried out in the clinical population of Croatian children and their mothers. The sample comprised 202 children (97 boys, and 105 girls), aged from 5 to 12 years, who were coming to the dental office for dental treatment. The children and their mothers were invited to come to a dental office for dental examination and assessment of dental fear and anxiety (DFA). The children were randomly selected for the study, and all of them were of good general health. All children's parents were fully informed of the nature and purpose of the study; hence, they gave their consent.

\section{Survey instruments and procedures}

A pediatric dentist and children obtained children's behavior ratings prior to treatment. Two scales were used for assessing a child's DFA: the Modified Child Dental Anxiety Scale - Faces Version (MCDAS-f) (13) and the Children's Fear Survey Schedule - Dental Subscale (CFSS-DS) (8). The Corah Dental Anxiety Scale (CDAS) was used $(2,3)$. Individual items of the scale were read out to a child and the child was asked to answer based on facial images on the MCDAS$\mathrm{f}$ scale prior to treatment. The children were shown five images of human faces. Subsequently, they were asked to choose one picture reflecting their feeling in the best way. The questionnaire was administered to each child before dental examination in the dental office after an explanation by one of the authors had been given. The children were not allowed to consult their mothers who were present during the completion of the questionnaire. Dental anxiety in mothers was as- nost i valjanost $(9-12)$. Kulturne razlike mogu biti važne u pouzdanosti nekih ljestvica za procjenu dentalnoga straha i anksioznosti kod djece $(11,13,14)$.

Wong i suradnici (15) razvili su Modified Child Dental Anxiety Scale (MCDAS) za uporabu kod djece u dobi od 8 do 15 godina. Ljestvica se temelji na Corah Dental Anxiety Scale (CDAS) i sadržava osam pitanja za procjenu anksioznosti u različitim dentalnim situacijama. Za rangiranje dentalne anksioznosti koristimo se petostupanjskom Likertovom ljestvicom. Ipak, MCDAS ljestvica nije prikladna za procjenu dentalne anksioznosti ako su djeca vrlo mala i još ne mogu dobro kognitivno funkcionirati.

Liječnice Howard i Freeman (13) izradile su Modified Child Dental Anxiety Scale - Faces Version (MCDAS-f) tako da su modificirale MCDAS - dodale su izvornoj numeričkoj ljestvici analognu ljestvicu lica. Dodavanje lica odgovarajućim brojevima omogućuje maloj i nervoznoj djeci dobro kognitivno funkcioniranje, pa je moguća odgovarajuća procjena dentalne anksioznosti. Evaluacija psihometrijskih svojstava ljestvice MCDAS-f potvrdila je da je riječ o pouzdanoj mjeri dentalne anksioznosti kod djece (13). Budući da se pouzdanost i valjanost takvih ljestvica treba testirati u različitim populacijama i kulturama, cilj ovoga rada je bio procijeniti pouzdanost i valjanost ljestvica CFSS-DS i MCDAS-f na uzorku hrvatske djece.

\section{Materijal i metode}

\section{Uzorak i njegova struktura}

Procjena dentalne anksioznosti provedena je na kliničkoj populaciji hrvatske djece i njihovih majki. Uzorak je obuhvaćao 202 djeteta (97 dječaka i 105 djevojčica) u dobi od 5 do 12 godina koja su dolazila u stomatološku ambulantu radi dentalnih zahvata. Djeca i njihove majke pozvani su da dođu u stomatološku ambulantu zbog pregleda i procjene dentalnoga straha i anksioznosti (DFA-e). Djeca su za istraživanje slučajno odabrana i sva su bila dobroga općeg zdravlja. Roditelji su bili potanko informirani o prirodi i svrsi istraživanja, nakon čega su potpisali pristanak.

\section{Korišteni instrumenti i postupci}

Rangiranje ponašanja djece prije liječenja dobiveno je od dječjeg stomatologa i djece. Korištene su dvije ljestvice za procjenu DFA-e - Modified Child Dental Anxiety Scale - Faces Version (MCDAS-f) (13) i Children's Fear Survey Schedule - Dental Subscale (CFSS-DS) (8). Corah Dental Anxiety Scale (CDAS) upotrijebljena je za procjenu dentalne anksioznosti kod majki u četirima različitim dentalnim situacijama $(2,3)$. Pojedina pitanja na ljestvici pročitana su djetetu i od njega se tražilo da prije liječenja odgovori na temelju izraza lica u MCDAS-f ljestvici. Naime, djeci je pokazano pet slika ljudskoga lica, a nakon toga tražilo se da odaberu onu koja najbolje odgovara njihovu osjećaju. Pitanja su postavljena prije stomatološkog pregleda i nakon objašnjenja doktora dentalne medicine. Tijekom ispunjavanja upitnika djeca se nisu mogla dogovarati s majkama. Dentalna anksioznost majki procijenjena je primjenom uobičajeno korište- 
sessed by the commonly used Corah's Dental Anxiety Scale (CDAS) measuring reactions to four different dental situations on a five-point scale $(2,3)$. Mothers were asked to fill in the CFSS-DS questionnaire for their children and the CDAS for themselves.

\section{Data analysis}

The differences in anxiety scores between the groups of children with cooperative behavior and anxiety with behavior management problem were tested by the Student's t-test or the Mann-Whitney test. All variables, such as total scores of scales, showing normal distribution, were analyzed by using parametric tests (t-test for independent samples). A nonparametric test (the Mann-Whitney test) was applied to the variables that did not show normal distribution. Different Spearman's rank correlations were computed to examine the relationship between three scales for dental fear and anxiety. The correlation coefficients between anxiety scores of self-reported measurements obtained for children and their mothers were calculated to establish the validity of scales $(7,17-20)$. The Cronbach's alpha value was calculated to assess the internal reliability of the scales. The index is based on the number of variables of the instrument and correlation coefficients among them since it is considered the most adequate index for assessing the reliability of the scales $(18,20,21)$.

\section{Results}

Differences in total scores of the Children's Fear Survey Schedule - Dental Subscale (CFSS-DS) regarding sexes and age were not statistically significant. Total scores of CFSS-DS were not normally distributed because the curve was skewed to the lower scores (Figure 1). The Mann-Whitney test was used to compare the obtained scores. All subjects were analyzed together and they displayed the average score of 30.84 $(\mathrm{n}=202, \mathrm{SD}=9,58)$ (Table 1). Children with dental anxiety and behavior management problems (BMP) displayed sig- ne ljestvice Corah's Dental Anxiety Scale (CDAS) koja mjeri reakcije u četirima različitim situacijama na petostupanjskoj ljestvici $(2,3)$. Od majki se tražilo da ispune CFSS-DS upitnik za svoje dijete i CDAS za sebe.

\section{Obrada podataka}

Razlike u vrijednostima skorova anksioznosti između skupina djece $s$ kooperativnim ponašanjem i one s problematičnim ponašanjem testirane su Studentovim t-testom ili Mann-Whitneyjevim testom. Sve varijable, kao ukupni skorovi ljestvica koje su pokazivale normalnu distribuciju, testirane su primjenom parametrijskog testa ( $\mathrm{t}$-test za nezavisne uzorke). Neparametrijski test (Mann-Whitneyjev) korišten je za varijable koje nisu pokazivale normalnu distribuciju. Spearmanove rank korelacije izračunate su za utvrđivanje odnosa između triju ljestvica za procjenu dentalnoga straha i anksioznosti. Za utvrđivanje valjanosti ljestvica izračunavani su korelacijski koeficijenti između skorova ljestvica dobivenih za djecu i njihove majke (7, 16 - 19). Vrijednosti Cronbachove alfe izračunavane su u svrhu procjene unutarnje pouzdanosti ljestvica. Indeks se temelji na broju varijabli instrumenta i korelacijskih koeficijenata među njima i smatra se najprikladnijim za procjenu pouzdanosti ljestvica $(17,19,20)$.

\section{Rezultati}

Razlike u ukupnim skorovima testa Children's Fear Survey Schedule - Dental Subscale (CFSS-DS) nisu bile značajne kad je riječ o spolu i dobi. Skorovi CFSS-DS-a nisu bili normalno distribuirani jer je krivulja bila pomaknuta prema onima nižima (slika 1.). Za usporedbu tih skorova korišten je Mann-Whitneyjev test. Svi ispitanici analizirani su zajedno i pokazivali su prosječan skor od 30,84 $(\mathrm{n}=202, \mathrm{SD}=9,58)$ (tablica 1.). Djeca s dentalnom anksioznošću i problemom u ponašanju (BMP-om) imala su značajno više prosječne sko-

\begin{tabular}{|c|c|c|c|c|c|}
\hline $\begin{array}{c}\text { Questionnaire / Scale • } \\
\text { Upitnik / ljestvica }\end{array}$ & Behaviour $\bullet$ Ponašanje & $\mathbf{n}$ & M & S. D. & $\mathbf{P}$ \\
\hline \multirow{3}{*}{$\begin{array}{l}\text { Child Fear Survey Schedule - Dental } \\
\text { Subscale (CFSS-DS) }\end{array}$} & Cooperative $\bullet$ Kooperativno & 158 & 28.46 & 7.69 & \multirow{3}{*}{$<0.001^{*}$} \\
\hline & Anxiety and BMP • Anksiozno i BMP & 44 & 39.36 & 10.85 & \\
\hline & Total $\bullet$ Ukupno & 202 & 30.84 & 9.58 & \\
\hline \multirow{3}{*}{$\begin{array}{l}\text { Modified Child Dental Anxiety Scale - } \\
\text { faces version (MCDAS-f) }\end{array}$} & Cooperative $\bullet$ Kooperativno & 158 & 20.01 & 4.58 & \multirow{3}{*}{$<0.001$} \\
\hline & Anxiety and BMP • Anksiozno i BMP & 44 & 28.07 & 4.69 & \\
\hline & Total $\bullet$ Ukupno & 202 & 21.77 & 5.67 & \\
\hline \multirow{3}{*}{ Corah Dental Anxiety Scale (CDAS)* } & Cooperative $\bullet$ Kooperativno & 158 & 10.05 & 3.05 & \multirow{3}{*}{$0.201^{\mathrm{a}}$} \\
\hline & Anxiety and BMP • Anksiozno i BMP & 44 & 11.05 & 3.89 & \\
\hline & Total $\bullet$ Ukupno & 202 & 10.27 & 3.27 & \\
\hline \multicolumn{6}{|c|}{$\begin{array}{l}\text { Legend } \bullet \text { Legenda: } \\
\mathrm{M}-\text { mean } \bullet \text { srednja vrijednost; S. D. }- \text { standard deviation } \bullet \text { standardna devijacija; BMP }- \text { behaviour management problem } \bullet \text { problem kontrole } \\
\text { ponašanja; }{ }^{\text {a }} \text { Mann-Whitney test } \bullet \text { Mann-Whitneyjev test, }{ }^{b} \text { t-test for independant samples } \bullet t \text {-test za nezavisne uzorke } \\
{ }^{*} \text { CDAS scores obtained for mothers of cooperative and BMP children } \bullet \text { CDAS skorovi dobiveni za majke kooperativne djece i djece s problemom u } \\
\text { ponašanju (BMP) }\end{array}$} \\
\hline
\end{tabular}




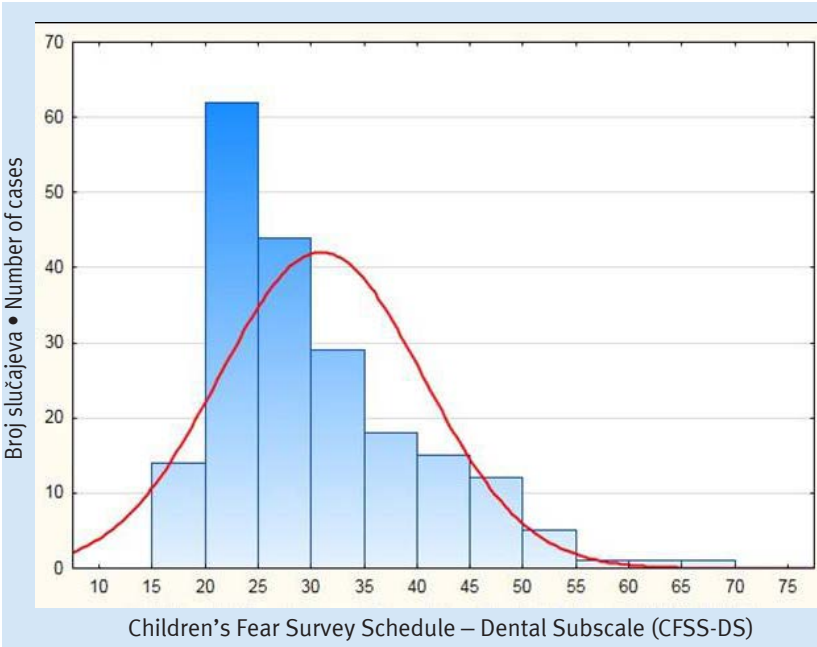

Figure 1. Distribution of CFSS-DS scores (scores are not normally distributed because majority of children scored low)

Slika 1. Distribucija CFSS-DS skorova (nisu normalno distribuirani jer većina djece pokazuje niske vrijednosti)

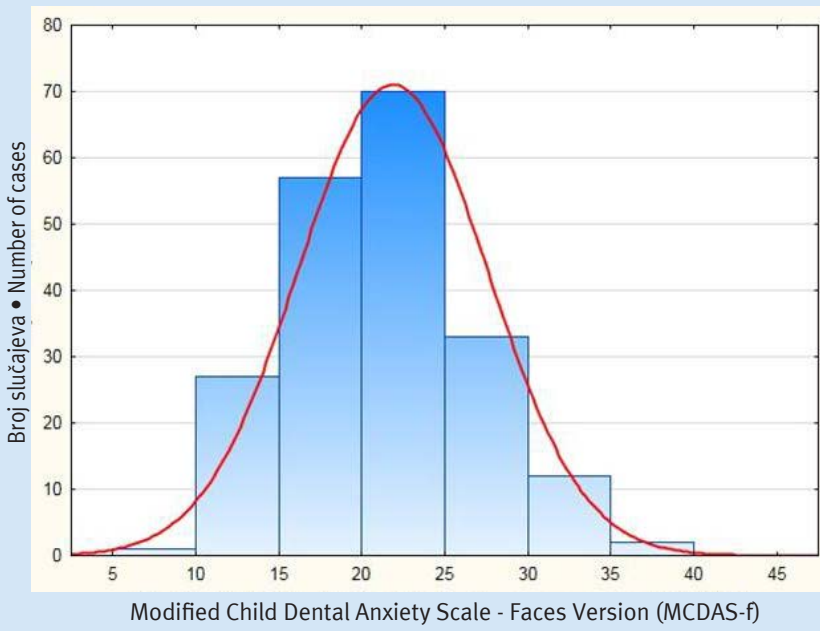

Figure 2. Normal distribution of Modified Child Dental Anxiety Scale Faces Version (MCDAS-f) scores

Slika 2. Normalna distribucija skorova Modified Child Dental Anxiety Scale - Faces Version (MCDAS-f) nificantly higher average score $(M=39.36)$ than the group of non-anxious children with cooperative behavior $(\mathrm{M}=28$. 46) $(\mathrm{P}<0.001)$.

The differences in a total score of MCDAS-f regarding sexes and age were not statistically significant and were on average 21. $77(n=202, S D=5.67)$. Significant differences between children with cooperative behavior and anxious children with BMP were obtained by comparison of MCDAS-f scores. The values of total MCDAS-f score showed the normal distribution in the present sample (Figure 2). The Student's independent t-test was used to test the significance of differences between the groups of children. While the cooperative children displayed an average MCDAS-f score of 20. 01, the children with BMP showed a significantly higher mean score $(M=28.07)$, which was statistically highly significant $(\mathrm{P}<0.001)$.

Mothers of children with cooperative behavior and children with BMP did not differ significantly regarding the level of dental anxiety assessed by the CDAS. The distribution of CDAS scores from 4 to 20 did not follow a normal distribution (the Kolmogorov-Smirnov test: $Z=2.082, p<0.001$ ). In the analysis of obtained scores, nonparametric tests were used.

Reliability of Children's Fear Survey Schedule - Dental Subscale (CFSS-DS) was assessed by the computation of Cronbach's $\alpha$ coefficient (Table 2). The resulting $\alpha$ value of 0.90 indicates a very high reliability of (CFSS-DS) test in assessing a child's fear and dental anxiety from dental treatment.

The reliability of the Modified Child Dental Anxiety Scale - faces version (MCDAS-f) was assessed by calculation of a Cronbach's $\alpha$ coefficient. All Cronbach a coefficients for each item and behavior of other items, if one item is deleted, are listed in Table 3, thus giving an insight into the uniformity of the items that make up the CFSS-DS test. The total value of Cronbach's $\alpha$ coefficient of 0.73 displayed acceptable reliability of the MCDAS-f in self-assessment of child's rove $(M=39,36)$ od skupine djece koja nisu bila anksiozna i surađivala su $(\mathrm{M}=28,46)(\mathrm{P}<0,001)$.

Razlike u ukupnim skorovima MCDAS-f testa, kad je riječ o spolu i dobi, nisu bile statistički značajne i njihova prosječna vrijednost bila je 21,77 ( $\mathrm{n}=202, \mathrm{SD}=5,67)$. Značajne razlike dobivene su usporedbom skorova MCDAS-f ljestvice između djece $s$ kooperativnim ponašanjem i one anksiozne s problemom u ponašanju (BMP-om). Vrijednosti ukupnih skorova MCDAS-f ljestvice pokazivale su normalnu distribuciju u analiziranom uzorku (slika 2). Za testiranje značajnosti razlika među skupinama djece korišten je Studentov test za nezavisne uzorke. Dok su kooperativna djeca pokazivala prosječan skor na MCDAS-f ljestvici od 20,01, djeca s BMP-om imala su znatno viši $(M=28,07)$, što je bilo statistički visoko značajno $(\mathrm{P}<0,001)$.

Majke djece $s$ kooperativnim ponašanjem i majke djece s BMP-em nisu se značajno razlikovale kad je riječ o razini dentalne anksioznosti procijenjene s pomoću CDAS testa. Razdioba CDAS-ovih skorova od 4 do 20 nije pokazivala normalnu distribuciju (Kolmogorov-Smirnovljev test: $\mathrm{Z}=$ 2,082, $\mathrm{p}<0,001)$. $U$ analizi dobivenih skorova korišteni su neparametrijski testovi.

Pouzdanost Children's Fear Survey Schedule - Dental Subscale (CFSS-DS) bila je procijenjena izračunavanjem Crombachova $\alpha$ koeficijenta (tablica 2.). Dobivena $\alpha$ vrijednost od 0,90 pokazuje vrlo visoku pouzdanost CFSS-DS testa za procjenu djetetova straha i dentalne anksioznosti od stomatološkog liječenja.

Pouzdanost ljestvice Modified Child Dental Anxiety Scale - faces version (MCDAS-f) procijenjena je izračunavanjem Crombachova $\alpha$ koeficijenta. Svi Crombachovi $\alpha$ koeficijenti za svaku česticu ponašanja, ako je jedna čestica izostavljena, nalaze se u tablici 3., što pruža uvid u sukladnost čestica koje čine CFSS-DS test. Ukupna vrijednost Cronbachova $\alpha$ koeficijenta od 0,73 pokazuje prihvatljivu pouzdanost MCDAS$\mathrm{f}$ testa u procjeni dentalne anksioznosti kod djece. Sve čestice Cronbachove alfe, ako je jedna čestica izostavljena, pokaziva- 
Table 2. Reliability of Children's Fear Survey Schedule-Dental Subscale (CFSS-DS)

Tablica 2. Pouzdanost ljestvice Children's Fear Survey Schedule-Dental Subscale (CFSS-DS)

Item-Total Statistics • Ukupna statistika čestica

\begin{tabular}{|c|c|c|c|}
\hline $\begin{array}{l}\text { Item of scale • } \\
\text { Čestica ljestvice }\end{array}$ & $\begin{array}{l}\text { Scale Mean if Item Deleted } \\
\text { Srednja vrijednost ljestvice ako je } \\
\text { čestica izostavljena }\end{array}$ & $\begin{array}{l}\text { Variance } \\
\text { Varijanca }\end{array}$ & $\begin{array}{c}\text { Cronbach's Alpha if Item Deleted • } \\
\text { Cronbachova alfa ako je čestica } \\
\text { izostavljena } \\
\alpha\end{array}$ \\
\hline Dentists $\bullet$ Doktor dentalne medicine (zubar) & 28.6584 & 75.6987 & 0.8910 \\
\hline Doctors $\bullet$ Doktor (liječnik) & 29.1188 & 82.0256 & 0.8984 \\
\hline Injections (shots) • Injekcija (ubod) & 27.9406 & 79.2900 & 0.9002 \\
\hline $\begin{array}{l}\text { Having somebody examine your mouth } \bullet \\
\text { Pregled zuba }\end{array}$ & 29.1584 & 79.3380 & 0.8941 \\
\hline $\begin{array}{l}\text { Having to open your mouth } \\
\text { Otvoriti i pokazati usta }\end{array}$ & 29.4604 & 83.0954 & 0.8978 \\
\hline $\begin{array}{l}\text { Having a stranger touch you } \bullet \\
\text { Dodir nepoznate osobe }\end{array}$ & 29.1931 & 83.2710 & 0.9006 \\
\hline $\begin{array}{l}\text { Having somebody look at you } \bullet \\
\text { Biti promatran od nekoga }\end{array}$ & 29.3119 & 86.8226 & 0.9061 \\
\hline The dentist drilling $\bullet$ Bušenje zuba & 27.7772 & 75.2984 & 0.8944 \\
\hline $\begin{array}{l}\text { The sight of the dentist drilling } \\
\text { Pogled na bušenje zuba }\end{array}$ & 28.3911 & 76.4881 & 0.8907 \\
\hline $\begin{array}{l}\text { The noise of the dentist drilling } \\
\text { Zvuk zubarske bušilice }\end{array}$ & 28.3960 & 76.3996 & 0.8909 \\
\hline $\begin{array}{l}\text { The dentist putting instruments in your mouth } \\
\text { Ulazak instrumentima u usta }\end{array}$ & 28.6436 & 77.4146 & 0.8906 \\
\hline Choking $\bullet$ Gušenje & 27.9356 & 81.3640 & 0.9027 \\
\hline $\begin{array}{l}\text { Having to go to the hospital } \bullet \\
\text { Odlazak u bolnicu (na liječenje) }\end{array}$ & 29.2426 & 86.2443 & 0.9131 \\
\hline $\begin{array}{l}\text { People in white uniforms } \\
\text { Ljudi u bijelim odorama }\end{array}$ & 29.4158 & 84.9506 & 0.9004 \\
\hline $\begin{array}{l}\text { Having the dentist clean your teeth } \bullet \\
\text { Čišćenja zuba kod doktora dentalne medicine }\end{array}$ & 29.0693 & 79.5076 & 0.8930 \\
\hline \multicolumn{3}{|l|}{ Cronbachova $\alpha$} & 0.9040 \\
\hline
\end{tabular}

Table 3. Reliability of Modified Child Dental Anxiety Scale - faces version (MCDAS-f)

Tablica 3. Pouzdanost ljestvice Modified Child Dental Anxiety Scale - faces version (MCDAS-f)

\begin{tabular}{|c|c|c|c|}
\hline \multirow{2}{*}{$\begin{array}{l}\text { Item of scale } \bullet \text { Čestica ljestvice } \\
\text { How do you feel ... } \\
\text { Kako se osjećaš ... }\end{array}$} & \multicolumn{3}{|c|}{$\begin{array}{l}\text { Item-Total Statistics } \bullet \text { Ukupna statistika ljestvice kada je } \\
\text { čestica izostavljena }\end{array}$} \\
\hline & $\begin{array}{l}\text { Scale Mean if Item } \\
\text { Deleted • } \\
\text { Prosjek }\end{array}$ & $\begin{array}{l}\text { Variance } \\
\text { Varijanca }\end{array}$ & $\begin{array}{l}\text { Cronbach's Alpha if } \\
\text { Item Deleted } \bullet \\
\text { Cronbahova alfa } \\
\text { ako je jedna čestica } \\
\text { izostavljena } \\
\alpha\end{array}$ \\
\hline $\begin{array}{l}\text {... about going to the dentist generaly? } \bullet \text {... kad trebaš ići doktoru dentalne medicine } \\
\text { (zubaru)? }\end{array}$ & 19.5792 & 25.4987 & 0.6866 \\
\hline$\ldots$ having your teeth looked at? $\bullet \ldots$ kad trebaš otići zubaru na pregled zuba? & 19.7030 & 25.8616 & 0.6819 \\
\hline $\begin{array}{l}\text {...having your teeth scraped and polished? } \bullet . . . \text { u vezi s čišćenjem i poliranjem tvojih } \\
\text { zuba? }\end{array}$ & 19.5891 & 25.9249 & 0.6969 \\
\hline$\ldots$ having an injection in the gum? $\bullet . . \mathrm{u}$ vezi $s$ dobivanjem injekcije $\mathrm{u}$ zubno meso? & 17.7871 & 26.5565 & 0.7016 \\
\hline$\ldots$ having a feeling? $\bullet . .$. ako je potrebna plomba na tvojem zubu? & 18.8911 & 24.6050 & 0.6920 \\
\hline$\ldots$ having a tooth taken out? $\bullet \ldots$ ako ti liječnik treba izvaditi zub? & 18.4851 & 25.3256 & 0.7122 \\
\hline$\ldots$ being put sleep to have treatment? $\bullet$... ako te treba uspavati da ti popravi zube? & 19.2030 & 25.5556 & 0.7305 \\
\hline $\begin{array}{l}\ldots \text { having a mixture of ,gas and air“ which will help you feel comfortable for } \\
\text { treatment but can not put you to sleep? } \bullet . . . \text { ako dobiješ masku za disanje s plinom i } \\
\text { osjećaš se ugodno tijekom liječenja, ali ti se ne spava? }\end{array}$ & 19.1337 & 25.4298 & 0.7045 \\
\hline \multicolumn{3}{|l|}{ Cronbachova $\alpha$} & 0.7280 \\
\hline
\end{tabular}

dental anxiety. All items of Cronbach's alpha showed a good internal consistency when one item was deleted. The assessment was based on the sum of total scores obtained by the le su dobru unutarnju konzistentnost. Procjena se temelji na zbroju svih skorova dobivenih s pomoću MCDAS-f instrumenta koji se teoretski kreću u rasponu od 8 do 40. 
Table 4. Reliability of Corah's Dental Anxiety Scale (CDAS)

Tablica 4. Analiza pouzdanosti testa Corah Dental Anxiety Scale (CDAS)

\begin{tabular}{|c|c|c|c|}
\hline \multirow[b]{2}{*}{ Item of scale $\bullet$ Čestica ljestvice } & \multicolumn{3}{|c|}{$\begin{array}{l}\text { Item-Total Statistics • Ukupna statistika ljestvice } \\
\text { kada je čestica izostavljena }\end{array}$} \\
\hline & $\begin{array}{l}\text { Mean } \bullet \\
\text { Prosjek }\end{array}$ & $\begin{array}{l}\text { Variance } \\
\text { Varijanca }\end{array}$ & $\begin{array}{l}\text { Cronbach's Alpha if } \\
\text { Item Deleted } \\
\alpha\end{array}$ \\
\hline $\begin{array}{l}\text { If you had to go to the dentist tomorrow, how would you feel about it? } \\
\text { Da sutra trebate otići doktoru dentalne medicine (zubaru), kako biste se osjećali? }\end{array}$ & 7.3564 & 7.1957 & 0.8797 \\
\hline $\begin{array}{l}\text { When you are in the dentist's chair waiting while he gets his drill ready to begin working on } \\
\text { your teeth, how do you feel? • Kako se osjećate dok čekate na zubarskom stolcu, a doktor } \\
\text { dentalne medicine (zubar) uzima bušilicu kako bi počeo raditi na vašem zubu? }\end{array}$ & 7.5248 & 5.3949 & 0.8390 \\
\hline $\begin{array}{l}\text { You are in the dentist's chair to have your teeth cleaned. While you are waiting and the dentist } \\
\text { is getting out the instruments which he will use to scrape your teeth around the gums, how } \\
\text { do you feel? } \bullet \text { Sjedite u zubarskom stolcu i čekate čišćenje zuba. Dok čekate, doktor dentalne } \\
\text { medicine (zubar) priprema instrumente kojima će očistiti zube oko zubnog mesa.., kako se } \\
\text { osjećate? }\end{array}$ & 7.9257 & 5.8999 & 0.8511 \\
\hline \multicolumn{3}{|l|}{ Cronbachova $\alpha$} & 0.8889 \\
\hline
\end{tabular}

MCDAS-f, which are theoretically in the range from 8 to 40 .

The reliability of the CDAS scale was assessed by means of a Cronbach's $\alpha$ coefficient that is based on the internal consistency of this instrument. Table 4 shows the Cronbach's $\alpha$ coefficient for each item of the scale and $\alpha$ score for the total CDAS scale. A total $\alpha$ coefficient was 0.89 , which means that there was a good internal consistency or good reliability of CDAS in the assessment of dental anxiety.

The Spearman's rank correlation coefficients showed a significant association between all three scales (Table 5). There was a high positive correlation between total scores of CFSSDS and MCDAS-f scales $(r=0,482 ; \mathrm{P}<0.01)$. The CDAS scores showed a significant positive correlation with CFSSDS $(r=0.223 ; P<0.01)$, while the correlation with MCDAS-f was lower but still significant $(\mathrm{r}=0.198 ; \mathrm{P}<0.01)$. All three scales showed good validity in assessing the child's behavior in dental situations.
Pouzdanost CDAS ljestvice procijenjena pomoću Cronbachova $\alpha$ koeficijenta temelji se na unutarnjoj konzistentnosti toga instrumenta. Tablica 4. pokazuje Cronbachove $\alpha$ koeficijente za svaku česticu ljestvice i $\alpha$ skor za ukupni CDAS. Ukupni $\alpha$ koeficijent bio je 0,89 , što znači da postoji dobra unutarnja konzistentnost i dobra pouzdanost ljestvice CDAS u procjeni dentalne anksioznosti.

Spearmanovi koeficijenti rank korelacija pokazali su značajnu povezanost između svih triju ljestvica (tablica 5). Ustanovljena je visoka pozitivna korelacija između ukupnih skorova ljestvica CFSS-DS i MCDAS-f $(\mathrm{r}=0,482$; $\mathrm{P}<0,01)$. CDAS-ovi skorovi pokazivali su značajnu pozitivnu korelaciju $s$ CFSS-DS-ovim $(r=0,223$; $\mathrm{P}<0,01)$, a korelacija $s$ MCDAS-f ljestvicom bila je niža, ali još uvijek značajna (r $=0,198 ; \mathrm{P}<0 ., 1)$. Sve tri ljestvice pokazale su dobru valjanost za procjenu ponašanja djeteta u različitim dentalnim situacijama.

\begin{tabular}{|c|c|c|c|c|c|}
\hline \multirow[t]{4}{*}{ Table 5.} & \multirow{4}{*}{$\begin{array}{l}\text { Spearman's correlation } \\
\text { coefficients between total } \\
\text { scores of three scales for } \\
\text { assessment of dental fear and } \\
\text { anxiety }(n=202)\end{array}$} & Variables $\bullet$ Varijable & CDAS & CFSS-DS & MCDAS-f \\
\hline & & CDAS & --- & $0.223^{* * *}$ & $0.198^{* * *}$ \\
\hline & & CFSS-DS & $0.223^{* * *}$ & -.- & $0.482^{* * *}$ \\
\hline & & MCDAS-f & $0.198^{* * *}$ & $0.482^{* * *}$ & --- \\
\hline Tablica 5. & $\begin{array}{l}\text { Spearmanovi korelacijski } \\
\text { koeficijenti između ukupnih } \\
\text { skorova triju ljestvica za } \\
\text { procjenu dentalnoga straha i } \\
\text { anksioznosti }(n=202)\end{array}$ & \multicolumn{4}{|c|}{$\begin{array}{l}\text { Legenda: CDAS - Corah Dental Anxiety Scale; CFSS-DS - Child Fear Survey Schedule-Dental Subscale; } \\
\text { MCDAS-f - Modified Child Dental Anxiety Scale - verzija s licima } \\
\text { Legend: CDAS - Corah Dental Anxiety Scale; CFSS-DS - Child Fear Survey Schedule-Dental Subscale; } \\
\text { MCDAS-f - Modified Child Dental Anxiety Scale - faces version } \\
* * * ~ P<0,01\end{array}$} \\
\hline
\end{tabular}

\section{Discussion and conclusions}

The research on dental fear and anxiety (DFA) in children in different countries has often revealed different results due to the specific cultural environment and differences in childrearing practices. Cultural and social differences vary considerably between countries. Likewise, normative data vary for

\section{Rasprava i zaključci}

Istraživanje dentalnoga straha i anksioznosti (DFA-e) kod djece u različitim zemljama često pokazuje različite rezultate zbog specifičnog kulturnog okružja i različite prakse u njihovu odgoju. Kulturne i socijalne razlike između zemalja znatno se razlikuju. Također variraju normativni podatci za svaku 
each measure in different populations. Since existing measures for evaluation of dental anxiety in children are not sensitive enough for the objective assessment, there is a need for development of new instruments (22). Such an instrument should be culturally sensitive and provide an objective assessment of dental anxiety in a specific population of children.

The average score of CFSS-DS in a Croatian sample in this study was 28.46 for non-anxious and cooperative children, while anxious children with BMP displayed a significantly greater score of 39.36 . The difference in the average score between cooperative children and children with BMP was statistically highly significant $(\mathrm{P}<0,001)$. The average score for Chinese children aged 6 to 10 years was $21.0 \pm 6$. 57. The mean scores of the CFSS-DS in different populations show considerable variations ranging from 22.1 to 37.0 $(9,14,23-26)$. We have not observed a significant difference between boys and girls in this study and both sexes were analyzed together. Ma et al. (14) also did not find significant differences in CFSS-DS mean scores among children of different ages.

Majstorovic et al. (27) examined dental anxiety in Croatian children using CDAS and CFSS-DS. In the sample of 165 children aged from 5 to 15 years, they established high reliability of CFSS-DS (Cronbach's $\alpha=0,8348$ ) and satisfactory reliability of CDAS with Cronbach's $\alpha$ of 0.7896 . A higher $\alpha$ coefficient obtained for CFSS-DS was attributed to children's difficulties with understanding of the CDAS scale. The CDAS has been frequently used because of its simplicity and because it can be completed in a shorter time.

The mean score of CFSS-DS for Croatian sample obtained by Majstorovic et al. (27) was 27. 02, which is similar to the results obtained in some other studies. The mean scores in studies based on the parental version of CFSS-DS varied from 23.1 to $29.7(1,23,26,27)$. A recent study with a modified version of CFSS-DS delivered very high internal consistency reliability (Cronbach's $\alpha=0,90)$ and high factorial validity (28).

A single measuring instrument that is currently available cannot measure multifactorial nature of dental anxiety adequately. Such a scale should comprise a child's age, developmental and cognitive aspects, and child-rearing conditions, psychosocial and economic conditions of a child's environment (22).

Various instruments have been used for the assessment of dental fear and anxiety in children. Out of many scales, the Children's Fear Survey Schedule - Dental Subscale (CFSSDS) became widely accepted as a highly reliable and valid instrument $(1,7,12,22)$. However, this scale does not provide a satisfactory application in very young children (13).

Folyan and Kolawole (22) have pointed to the need for the development of an instrument that would enable young children to pick and point out a picture that illustrates the perceived child's emotion and anxiety. Such an instrument would be easily administered and used in children with limited cognitive and linguistic abilities. Howard and Freeman (13) have developed such an instrument. This instrument was scheduled to measure dental fear and anxiety in young children and is known as the MCDAS-f. They (13) proposed mjeru u različitim populacijama. Budući da postojeće mjere za evaluaciju dentalne anksioznosti kod djece nisu dovoljno osjetljive za objektivnu procjenu, potrebni su novi instrumenti (21) koji bi trebali biti kulturološki osjetljivi i osigurati objektivnu procjenu dentalne anksioznosti u specifičnoj populaciji djece.

Prosječan skor CFSS-DS testa u hrvatskom uzorku u ovom je istraživanju iznosio 28,46 za neanksioznu i kooperativnu djecu, a anksiozna djeca s problemom u ponašanju (BMP-om) pokazivala su značajno veći skor - 39,36. Razlika u prosječnim skorovima između kooperativne djece i djece s BMP-om bila je statistički visoko značajna $(\mathrm{P}<0,001)$. Prosječan skor za kinesku djecu u dobi od 6 do 10 godina bio je 21,0 $\pm 6,57$. Utvrđeno je da srednja vrijednost skora CFSS-DS u različitim populacijama pokazuje znatne varijacije u rasponu od 22,1 do 37,0 (9, 14, 22 - 25). Mi nismo uočili značajne razlike između dječaka i djevojčica u ovom istraživanju, pa su oba spola analizirana zajedno. Ma i suradnici (14) također nisu našli značajne razlike u CFSS-DS skorovima između djece različite dobi.

Majstorović i suradnici (26) istraživali su dentalnu anksioznost kod hrvatske djece primjenom ljestvica CDAS i CFSS-DS. Na uzorku od 165 djece u dobi od 5 do 15 godina oni su utvrdili visoku pouzdanost CFSS-DS instrumenta (Cronbachova $\alpha=0,8348)$ i zadovoljavajuću pouzdanost CDAS ljestvice s Cronbachovim a koeficijentom od 0,7896. Viši $\alpha$ koeficijent dobiven za CFSS-DS pripisan je poteškoći djece u razumijevanju te ljestvice. CDAS je često korišten zbog jednostavnosti i kratkog vremena potrebnog za ispunjavanje.

Srednja vrijednost CFSS-DS testa za hrvatski uzorak koju su dobili Majstorović i suradnici (26) bila je 27,02, što je vrlo blizu rezultatima dobivenima u nekim drugim studijama. Srednja vrijednost skora u studijama temeljenima na roditeljskoj verziji CFSS-DS-a varira od 23,1 do 29,7 (1, 22, 25). Novija studija $s$ prilagođenom verzijom ljestvice CFSS-DS omogućila je vrlo visoku unutarnju konzistentnost i pouzdanost (Cronbachova $\alpha=0,90$ ) te visoku faktorsku valjanost (27).

Multifaktorska priroda dentalne anksioznosti ne može se odgovarajuće mjeriti s pomoću jednoga mjernog instrumenta koji je danas dostupan. Takva bi ljestvica morala obuhvaćati djetetovu dob, razvojne i kognitivne aspekte, uvjete odgoja, psihosocijalne i ekonomske uvjete te djetetovu okolinu (21).

Za procjenu djetetova dentalnog straha i anksioznosti korišteni su različiti instrumenti. Od mnogobrojnih ljestvica, Children's Fear Survey Schedule - Dental Subscale (CFSSDS) postala je široko prihvaćena kao visoko pouzdan i valjan instrument $(1,7,12,21)$. Ipak, ni ona ne osigurava zadovoljavajuću primjenu kad je riječ o vrlo maloj djeci (13).

Liječnice Folyan i Kolawole (21) upozorile su da je potreban instrument koji će omogućiti maloj djeci da odaberu sliku i pokažu svoje emocije i anksioznost. Takav instrument mogao bi se jednostavno primijeniti u slučaju djece s ograničenim kognitivnim i lingvističkim sposobnostima. Jedan takav izrađen za mjerenje dentalnoga straha i anksioznosti kod male djece i poznat je kao MCDAS-f ljestvica, a izradile su ga liječnice Howard i Freeman (13). One (13) su predložile no- 
a new version of the MCDAS by adding faces to each item of a child's responses in the questionnaire. This new instrument was more suitable for very young and nervous children. Its main advantages are that it is simple, easy for administration and suitable for use in young children. Patients can point the face that expresses their feeling at this moment (13).

So far, there have not been any studies on Croatian children to establish reliability and validity of the MCDAS-f scale. The confident measures for assessing a child's dental fear and anxiety should be simple for application and understandable to young children to provide an objective assessment of their dental anxiety (22). The MCDAS-f provides administration in children with limited cognitive and linguistic abilities and is suitable for both young and older children.

The MCDAS-f total score for children with cooperative behavior in the present sample $(M=20,01)$ was almost the same as the normative score $(M=19.81)$ obtained by Howard and Freeman (13). The values of a total MCDAS-f score showed normal distribution in the present sample, and the Student's t-test was used to test differences in total scores between cooperative non-anxious and anxious children with BMP. The result showed that anxious children with BMP displayed significantly higher MCDAS-f score than non-anxious children (28. 07 vs. 20.01 ; $\mathrm{P}<0.001)$. The MCDAS-f in Howard and Freeman (13) study showed good validity and reliability with a Cronbach's alpha of 0.82 .

In the present study, the CFSS-DS showed a high level of reliability with Cronbach's $\alpha$ of 0.90 . The internal consistency of this instrument was reported to range from 0.83 to $0.92(7,10-12,14,27,28)$. Regarding the issue of reliability, Streiner et al. (17) pointed out that measuring scales with high reliability have to be used. Cronbach's $\alpha$ of 0.70 can be considered the minimum value of reliability. A test with a reliability of 0.80 would require a 25 per cent increase in sample size, and reliability of 0.70 would require a 43 per cent larger number of subjects (22).

The CDAS for the assessment of mothers' dental anxiety in the present study showed good internal consistency reliability (Cronbach's $\alpha=0.89$ ) with an average score of 10.05. The level of dental anxiety in mothers of non-anxious and cooperative children did not differ significantly from the mean score of mothers whose children displayed high dental anxiety and behavior management problem.

Karras (17) has pointed out that the validity coefficient of a test equals the coefficient of correlation between the test and its reference criterion. Although the coefficients of 0.80 are considered desirable, the significant coefficients could be based on the p-values of the correlation. Therefore, even much lower values of correlations could mean that a particular test is highly valid.

The Spearman's rank correlation coefficients in the present study showed significant association between CFSS-DS scores and MCDAS-f $(r=0.482 ; \mathrm{P}<0.01)$ (Table 6). The significant correlations suggest good validity of MCDAS- $f$ in a Croatian sample of children. The children's ratings on the CFSS-DS and MCDAS-f showed significant correlations with their mothers' level of dental anxiety assessed by CDAS vu verziju MCDAS-a tako da su dodale lica odgovoru na svako pitanje u upitniku. Taj novi instrument bio je prikladniji za vrlo malu i nervoznu djecu. Njegova glavna prednost jest što je jednostavan, lagan za primjenu i prikladan za vrlo malu djecu. Pacijenti mogu pokazati lice koje u tom trenutku izražava njihove osjećaje (13).

Dosad nije bilo istraživanja hrvatske djece kako bi se utvrdila pouzdanost i valjanost MCDAS-f ljestvice. Pouzdane mjere za procjenu djetetova dentalnog straha i anksioznosti trebale bi biti jednostavne za primjenu i razumljive maloj djeci kako bi se osigurala objektivna procjena njihove dentalne anksioznosti (21). MCDAS-f ljestvica omogućuje primjenu kod djece s ograničenim kognitivnim i lingvističkim sposobnostima, a prikladna je i za malu i za veću djecu.

Ukupan MCDAS-f skor za djecu s kooperativnim ponašanjem u ovom uzorku $(M=20,01)$ bio je gotovo isti kao i normativni skor $(M=19,81)$ koji su dobile Howardova i Freemanova (13). Vrijednost ukupnoga MCDAS-f skora pokazuje normalnu distribuciju u ovom istraživanju, pa je korišsten Studentov t-test za testiranje razlika u ukupnim skorovima između kooperativne i neanksiozne djece te one anksiozne s BMP-om. Rezultat pokazuje da anksiozna djeca s BMP-om pokazuju značajno veći skor MCDAS-f od one neanksiozne (28,07 prema 20,01; $\mathrm{P}<0,001)$. MCDAS-f ljestvica u istraživanju Howardove i Freemanove (13) pokazala je dobru valjanost i pouzdanost $s$ Cronbachovim $\alpha$ koeficijentom od 0,82 .

U ovom istraživanju CFSS-DS ljestvica pokazala je visoku razinu pouzdanosti s Cronbachovim $\alpha$ koeficijentom od 0,90 . Utvrđeno je da se unutarnja konzistentnost toga instrumenta kreće od 0,83 do $0,92(7,10-12,14,26)$. Kad je rije o pouzdanosti, Streiner i suradnici (17) ističu da se trebaju primijenjivati mjerne ljestvice $s$ visokom pouzdanošću. Cronbachova $\alpha$ od 0,70 može se smatrati minimalnom vrijednošću pouzdanosti. Test s pouzdanošću od 0,80 zahtijevao bi 25 posto povećanja veličine uzorka, a pouzdanost od 0,70 tražila bi 43 posto više ispitanika (21).

CDAS ljestvica za procjenu dentalne anksioznosti majki u ovom istraživanju pokazala je dobru unutarnju konzistentnost i pouzdanost (Cronbachova $\alpha=0,89$ ) s prosječnim skorom od 10,05. Razina dentalne anksioznosti majki neanksiozne i kooperativne djece nije se značajno razlikovala od srednjih vrijednosti skora majki čija su djeca pokazivala visoku dentalnu anksioznost i imala su probleme u ponašanju.

Karras (16) ističe da je koeficijent valjanosti testa jednak koeficijentu korelacije između testa i njegova referentnog kriterija. Premda se smatra da su koeficijenti od 0,80 poželjni, značajnost koeficijenta može se temeljiti na p-vrijednostima q korelacija. Prema tome, čak mnogo niže vrijednosti korelacija mogu značiti značajnu valjanost nekog testa.

Spearmanovi koeficijenti rank korelacija u ovom istraživanju pokazali su značajnu povezanost između skorova CFSS-DS i MCDAS-f ljestvica $(r=0,482 ; \mathrm{P}<0,01)$ (tablica 6.). Značajne korelacije upućuju na dobru valjanost MCDAS-f ljestvice na hrvatskom uzorku djece. Rangiranje djece s pomoću CFSS-DS i MCDAS-f ljestvica pokazalo je značajne korelacije s razinom anksioznosti njihovih majki koja je bila procijenjena CDAS ljestvicom $(r=0,223$, odnosno $r$ 
$(\mathrm{r}=0.223$ and $\mathrm{r}=0.198 ; \mathrm{P}<0.01$, respectively $)$, thus suggesting the influence of mothers' anxiety on their children's dental anxiety. The MCDAS-f was significantly correlated with the CFSS-DS regarded as a "gold standard" for assessing child dental anxiety (13). A high correlation between the CFSS-DS and CDAS scales was found in other studies (6, 30). They have concluded that these scales provide good tools for the assessment of dental anxiety in children.

A high value of Cronbach's alpha $(\mathrm{a}=0.8641)$ led the authors of this paper to conclude that two rating scales provide highly reliable instruments for the assessment of children's dental fear and anxiety in a dental office before dental procedures. The MCDAS-f and CFSS-DS showed a significant correlation and represent valid and suitable assessment tools for the assessment of dental fear in children. The MCDAS-f scale can be used in young children with limited cognitive and language skills. It is a suitable, simple to administer and reliable instrument for assessing dental anxiety in young children.

\section{Conflict of interest}

None declared
$=0,198 ; \mathrm{P}<0,01)$, što upozorava na utjecaj majčine anksioznosti na dentalnu anksioznost njihove djece. MCDAS-f pokazivao je značajnu korelaciju s CFSS-DS ljestvicom koja se smatra zlatnim standardom za procjenu dentalne anksioznosti kod djece (13). Visoka korelacija između skorova ljestvica CFSS-DS i CDAS nađena je i u drugim studijama $(6,28)$. Njihovi su autori zaključili da su te ljestvice dobri instrumenti za procjenu dentalne anksioznosti kod djece.

Visoka vrijednost Cronbachove alfe $(\mathrm{a}=0,8641)$ dovodi do zaključka u ovom istraživanju da obje ljestvice omogućuju visoko pouzdane instrumente za procjenu dentalnoga straha i anksioznosti kod djece u stomatološkoj ordinaciji prije liječenja. MCDAS-f i CFSS-DS ljestvice pokazuju značajnu korelaciju te su valjan i prikladan instrument za procjenu dentalnoga straha kod djece. MCDAS-f ljestvica može se primijeniti kad je riječ o maloj djeci s ograničenim kognitivnim i lingvističkim sposobnostima zato što je prikladna, jednostavna za primjenu i pouzdan je instrument za procjenu dentalne anksioznosti male djece.

\section{Sukob interesa}

Autori nisu bili u sukobu interesa.
Sažetak

Uvod: Djeca s visokom dentalnom anksioznošću nekooperativno se ponašaju tijekom dentalnih postupaka. Zbog toga su u uporabi različiti upitnici za procjenu prevalencije dentalnoga straha i anksioznosti (DFA-e) kod djece, ali potrebno je procijeniti njihovu pouzdanost i valjanost u različitim populacijama. Svrha rada: Željela se procijeniti pouzdanost i valjanost dviju ljestvica za procjenu dječjega dentalnog straha i anksioznosti na uzorku hrvatske djece. Materijal i postupci: Uzorak je obuhvaćao 202 para djece i njihovih majki (97 dječaka i 105 djevojčica) u dobi od 5 do 12 godina. Korištene su dvije ljestvice za procjenu dentalnoga straha i anksioznosti: Children's Fear Survey Schedule - Dental Subscale (CFSS-DS) i Modified Child Dental Anxiety Scale - face version (MCDAS-f). Corah's Dental Anxiety Scale (CDAS) primijenjena je na uzorku majki ispitanika. Uspoređeni su srednji skorovi kooperativne djece i djece s problemima u ponašanju (BMP-om). Rezultati: Djeca koja su surađivala pokazivala su značajno niže CFSS-DS skorove od djece s BMP-om ( $M=28,46$ prema $M=39,36 ; P<0,001)$. Prosječna vrijednost MCDAS-f bodova bila je značajno viša kod djece $s$ BMP-om negoli kod one kooperativne ( $M=28,07$ prema 20, 01; $P<0,001)$. CFSS-DS pokazao je visoku unutarnju konzistenciju (Cronbachova $\alpha=0,90$ ), a unutarnja konzistentnost MCDAS-f ljestvice bila je dobra $(\alpha=0,73)$. Majčin CDAS imao je dobru unutarnju konzistentnost $(\alpha=0,89)$. Korelacije s ostalim mjerama dentalnoga straha i anksioznosti pokazale su dobru valjanost obiju ljestvica. Korelacija između CFSS-DS i MCDAS-f ljestvica bila je visoko značajna $(r=0,482 ; P<0,01)$ kao i korelacije CFSS-DS-a i MCDAS-f ljestvica s CDAS skorovima $(r=0,223 ; P<0,01$, odnosno $r=0,198 ; P<0$, 01). Zaključak: Dobiveni rezultati pokazuju na uzorku hrvatske djece da su obje ljestvice (CFSS-DS i MCDAS-f) pouzdane i valjane za mjerenje dentalnoga straha i anksioznosti kod djece. MCDAS-f ljestvica imala je nešto nižu unutarnju konzistenciju, ali je prikladna za primjenu kod male djece. MCDASf ljestvica također je jednostavna za primjenu.
Zaprimljen: 17. prosinca 2019.

Prihvaćen: 2. veljače 2020.

Adresa za dopisivanje dr. Tomislav Śkrinjarić Sveučilište u Zagrebu Stomatološki fakultet Gundulićeva 5 10000 Zagreb tskrinjaric@sfzg.hr

\section{Ključne riječi}

dijete; strah; dentalna tjeskoba; upitnik o zdravlju bolesnika; provjera valjanosti

\section{References}

1. Klingberg G, Broberg A. Dental fear/anxiety and dental behaviour management problems in children and adolescents: a review of prevalence and concomitant psychological factors. Int J Paediatr Dent. 2007 Nov;17(6):391-406.

2. Corah NL. Development of a dental anxiety scale. J Dent Res. 1969 Jul-Aug;48(4):596.

3. Corah NL, Gale EN, Illig SJ. Assessment of a dental anxiety scale. I Am Dent Assoc. 1978 Nov;97(5):816-9.

4. Bhattacharya S, Kaur R, Kaur S, Ali SA. Validity and reliability of a questionnaire: a literature review. Chronicles of Dental Research 2017; Vol6(2):16-22.

5. Peretz B, Nazarian Y, Bimstein E. Dental anxiety in a students' paediatric dental clinic: children, parents and students. Int Paediatr Dent. 2004 May;14(3):192-8.

6. Kowalińska-Kania M, Nowak D, Waliczek M, Skowron M, Roczniak W, Żelazko A, Nowak PG. Numerical scale for anxiety assessement in 10-15 years children undergoing dental treatment. Dent Med Probl 2015;52(3):309-315.

7. Aartman IH, van Everdingen T, Hoogstraten J, Schuurs AH. Selfreport measurements of dental anxiety and fear in children: a critical assessment. ASDC J Dent Child. 1998 Jul-Aug;65(4):252-8, 229-30.

8. Cuthbert MI, Melamed BG. A screening device: children at risk for dental fears and management problems. ASDC J Dent Child. 1982 Nov-Dec;49(6):432-6.

9. Klingberg G. Reliability and validity of the Swedish version of the Dental Subscale of the Children's Fear Survey Schedule, CFSSDS. Acta Odontol Scand. 1994 Aug;52(4):255-6.

10. Nakai Y, Hirakawa T, Milgrom P, Coolidge T, Heima M, Mori Y, Ishihara C, Yakushiji N, Yoshida T, Shimono T. The children's fear survey scheduledental subscale in Japan. Community Dent Oral Epidemiol. 2005 Jun;33(3):196-204. 
11. Singh P, Pandey R K, Nagar A, Dutt K. Reliability and factor analysis of children's fear survey schedule-dental subscale in Indian subjects. J Indian Soc Pedod Prev Dent. 2010 JulSep;28(3):151-5.

12. Bajrić E, Kobašlija $S$, Jurić $H$. Reliability and validity of Dental Subscale of the Children's Fear Survey Schedule (CFSS-DS) in children in Bosnia and Herzegovina. Bosn J Basic Med Sci. 2011 Nov;11(4):214-8.

13. Howard KE, Freeman R. Reliability and validity of a faces version of the Modified Child Dental Anxiety Scale. Int J Paediatr Dent. 2007 Jul;17(4):281-8

14. Ma L, Wang M, Jing Q, Zhao J, Wan K, Xu Q. Reliability and validity of the Chinese version of the Children's Fear Survey ScheduleDental Subscale. Int J Paediatr Dent. 2015 Mar;25(2):110-6.

15. Wong HM, Humphris GM, Lee GTR. Preliminary validation and reliability of the Modified Child Dental Anxiety Scale. Psychol Rep. 1998 Dec;83(3 Pt 2):1179-86.

16. Zukanović A, Habibović J, Habibović E, Ajanović M, Bajrić E. Evaluation of dental fear and anxiety in displaced persons in Bosnia and Herzegovina. Acta Stomatol Croat. 2018 Jun;52(2):140-147.

17. Karras DJ. Statistical Methodology: 11. Reliability and Validity Assessment in Study Design, Part B. Acad Emerg Med. 1997; 4(2):144-149.

18. Streiner DL, Norman GR, Cairney J. Health Measurement Scales. A practical guide to their development and use. 5th ed. Oxford: Oxford University Press; 2015.

19. Appukuttan D, Datchnamurthy M, Sherley P. Deborah MS Hirudayaraj GJ, Tadepalli A, Victor DJ. Reliability and validity of the Tamil version of Modified Dental Anxiety Scale. J Oral Sci. 2012;54(4):313-20.

20. Bolarinwa OA. Principles and methods of validity and reliability testing of questionnaires used in social and health science researches. Niger Postgrad Med J. 2015 Oct-Dec;22(4):195-201.
21. Anastasiadou SD. Reliability and validity testing of a new scale for measuring attitudes toward learning statistics with technology. Acta Didact Napocensia. 2011; 4(1):1-10.

22. Folayan MO, Kolawole KA. A critical appraisal of the use of tools for assessing dental fear in children. Afr J Oral Health. 2004;1(1):5463.

23. Milgrom $P$, Fiset $L$, Melnick S, Weinstein $P$. The prevalence and practice management consequences of dental fear in a major US city. J Am Dent Assoc. 1988 May;116(6):641-7.

24. Alvesalo I, Murtomaa H, Milgrom P, Honkanen A, Karjalainen M, Tay KM. The Dental Fear Survey Schedule: a study with Finnish children. Int J Paediatr Dent. 1993 Dec;3(4):193-8.

25. ten Berge M, Veerkamp JS, Hoogstraten J, Prins PJ. On the structure of childhood dental fear, using the Dental Subscale of the Children's Fear Survey Schedule. Eur J Paediatr Dent. 2002 Jun;3(2):73-8.

26. Lee C-Y, Chang Y-Y, Huang S-T. Prevalence of dental anxiety among 5- to 8-year old Taiwanese children. J Public Health Dent. 2007 Winter;67(1):36-41.

27. Majstorovic M, Veerkamp JSJ, Skrinjaric I. Reliability and validity of measures used in assessing dental anxiety in 5- to 15-year-old Croatian children. Eur J Paediatr Dent. 2003 Dec;4(4):197-202.

28. Haliti F, Juric $H$. The relationship between dental trauma, anxiety and aggression behavior in 7 to14 year old children in Kosovo. Acta Stomatol Croat. 2017 Mar;51(1):3-12.

29. Bajrić E, Kobašlija S, Huseinbegovic A, Selimovic-Dragas $M$, Arslanagic Muratbegovic A, Markovic N. Reliability and validity of the Modified Version of Children's Fear Survey Schedule Dental Subscale in 9-12 Years Old Schoolchildren in Bosnia and Herzegovina. Med Arch. 2018 Jun;72(3):192-196.

30. Coric A, Banozic A, Klaric M, Vukojevic K, Puljak L. Dental fear and anxiety in older children: an association with parental dental anxiety and effective pain coping strategies. J Pain Res. 2014 Aug 20;7:515-21. 